\title{
Low-dimensional moderators at ESS and compact neutron sources
}

\author{
Luca Zanini1 ${ }^{1,}$, Esben Klinkby ${ }^{12}$, Ferenc Mezei ${ }^{1}$, and Alan Takibayev ${ }^{1}$ \\ 'European Spallation Source ESS ERIC, P.O Box 176, Lund, Sweden \\ ${ }^{2}$ DTU Nutech, Technical University of Denmark, Roskilde, Denmark
}

\begin{abstract}
Low-dimensional moderators were designed for the European Spallation Source (ESS), and the same concepts can be applied to compact sources. At ESS, quasi two-dimensional (2D) high-brightness moderators will serve all the instruments of the initial suite. The design of the moderators is influenced by several factors, such as the layout of the facility, the requirements for beam extraction, and the number of instruments; these constraints led to the choice of the 2D "butterfly" moderator for ESS. In an acceleratorbased compact source, such moderators can be designed in an even more efficient way than for high-power sources, taking advantage of the lower heat loads and of the more compact arrangement of the targetmoderator system, which gives more freedom in the optimization of the geometrical setup. Some promising design options have been explored.
\end{abstract}

\section{Introduction}

The neutron moderators designed for ESS are based on the low-dimensional moderator principles, which are described in Ref. [1] and several references therein. The design of the ESS moderators has been finalized and details are given in Ref. [1]. Since the early development of these moderators, we explored the possibility to use them in research reactors [2], and more recently in compact sources [3]. In [3] it was suggested that the use of low-dimensional moderators is more efficient (in terms of brightness per fast neutron produced in the target) in compact sources than for high-power facilities such as ESS. In this work we further explore the applicability of such moderators in compact neutron sources.

\section{Main features of the ESS moderators}

ESS will be the most powerful spallation neutron source in the world, with an average beam power of $5 \mathrm{MW}$ and peak power of $125 \mathrm{MW}$. The facility is designed with 42 beamports surrounding the moderators, covering an angle of 240 degrees, with an average angular spacing between beamports of 6 degrees. Such a grid of beamports is intended to give flexibility in positioning instruments over the lifetime of the facility. All the instruments in the initial scope of ESS will point to the moderators placed at the top of the tungsten spallation target. The requirement that the moderators should provide a bispectral (thermal and cold) neutron spectrum for all the beamports arranged in two sectors of 120 degrees each had a strong influence on the design of the moderator shape: the so-called "butterfly" design was the best identified configuration to fulfil this requirement. The height of the moderator was set to $3 \mathrm{~cm}$, after an optimization process that involved all the initial instruments in the ESS suite, and considered the moderator brightness as well as the brightness transfer from the guides to the sample. Details on the optimization process are given in Refs. [1,4]. Some upgrade possibilities are discussed in Ref. [1].

\section{Low-dimensional moderators for compact sources}

The energy of the neutrons emitted from the target depends on the primary beam energy; for compact sources, see Ref. [5]: for lower proton energies, and consequently, lower neutron energies, a more compact system can be designed, i.e., with a smaller reflector and a thinner premoderator. Compared to a spallation source, in a compact neutron source the neutron production takes place in a smaller region and this can result in a gain in performance, as the moderator can be placed closer to the neutron production region. This advantage can be exploited very effectively with low-dimensional moderators, especially quasi one-dimensional (1D) moderators. In the case of ESS, the neutron production region occupies a surface close to the one of the (optimized) butterfly moderator [1], which makes it particularly suitable for the use of 2D moderators, giving the possibility to serve a large number of instruments with a high-brightness moderator.

"Corresponding author: luca.zanini@esss.se 
An additional advantage for compact sources is due to the lower power, which allows moving the optics closer to the moderator. In the case of ESS the moderator thickness $(3 \mathrm{~cm})$ was chosen as the best compromise between source brightness and brightness transfer by the neutron optics [4]. Based on brightness considerations only, a thinner moderator would have been better performing [1]. For a compact source, it should be possible to move the optics closer to the moderator, and therefore to choose a thinner moderator than used at ESS.

For application to compact sources, in order to maximize the brightness, we considered in this work the quasi 1dimensional tube moderators, i.e., moderators with a cross section of a few $\mathrm{cm}^{2}$, and with a length of about $20 \mathrm{~cm}$, consisting of aluminium vessels filled with pure parahydrogen at $20 \mathrm{~K}$. Such moderators have a strong directional emission of cold neutrons [1], which will limit the number of beamlines that can be installed.

We did not consider beam transport aspects in this work, but focused only on the brightness of the source.

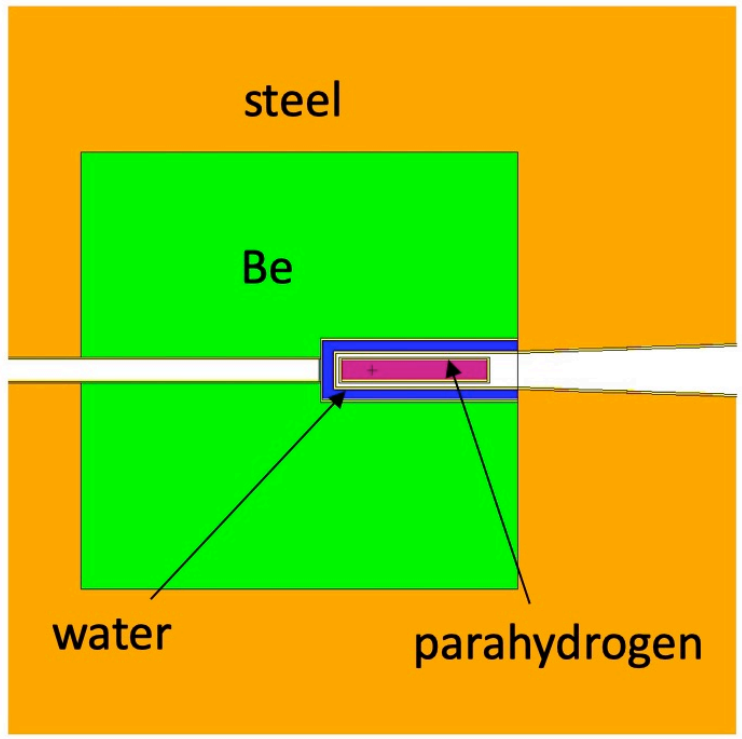

Fig. 1. 1-target geometry configuration.

\subsection{Source configurations}

We considered a neutron source from a $13 \mathrm{MeV}$ proton beam impinging on a 2-mm thick Be target. The choice of the energy was taken considering the example of the LENS compact source [6]: the $13 \mathrm{MeV}$ energy is at the threshold of production of ${ }^{7} \mathrm{Be}$ and ${ }^{3} \mathrm{H}$ in the target, thus limiting the activation of the target and simplifying the maintenance procedures.

We considered two possible geometry setups. In the first configuration (Fig. 1), the beryllium target is placed at the center of a beryllium reflector. A cylindrical aluminium tube with $3 \mathrm{~cm}$ diameter, filled with parahydrogen is surrounded by a water premoderator and is placed right after the target. We note that in this basic setup, fast neutrons would be directly seen in the beam extraction channels, a drawback that could be easily avoided by placing the moderator at an angle with respect to the proton beam line. The considered geometry is however suitable for the study of the performance of a tube moderator in such a compact source. In the second configuration (Fig. 2), the proton beam is split and sent to two beryllium targets. The moderator is placed between the two targets. In our example, the moderator is parallel to the proton beam line. A different configuration would be with the moderator perpendicular to the proton beam; this would have the advantage of doubling the neutron beam lines served by the moderator.

The neutron beam extraction channel is in both cases of conical shape, which could in principle accommodate the extraction of neutrons for more than one instrument. However, for simplicity we studied only the brightness at the center of the channel.

The dimensions of the target surface, irradiated by protons, are small $\left(1.5 \times 3 \mathrm{~cm}^{2}\right)$. To run at power levels of the order of tens of $\mathrm{kW}$ a larger target would be needed. A larger target surface will result in a decrease in performance, as shown in Section 3.6. Additionally, no engineering details (such as the cooling of target and reflector, and the presence of water and hydrogen pipes) are considered in this model. The reflector is a cube of $60 \mathrm{~cm}$ side. Simulations were performed with MCNPX 2.7.0 [7,8]. Neutron production cross sections in Be from $\mathrm{ENDF} / \mathrm{B}-\mathrm{VII}$ were used. The figure of merit for the design optimization was the integrated cold brightness below $5 \mathrm{meV}$.

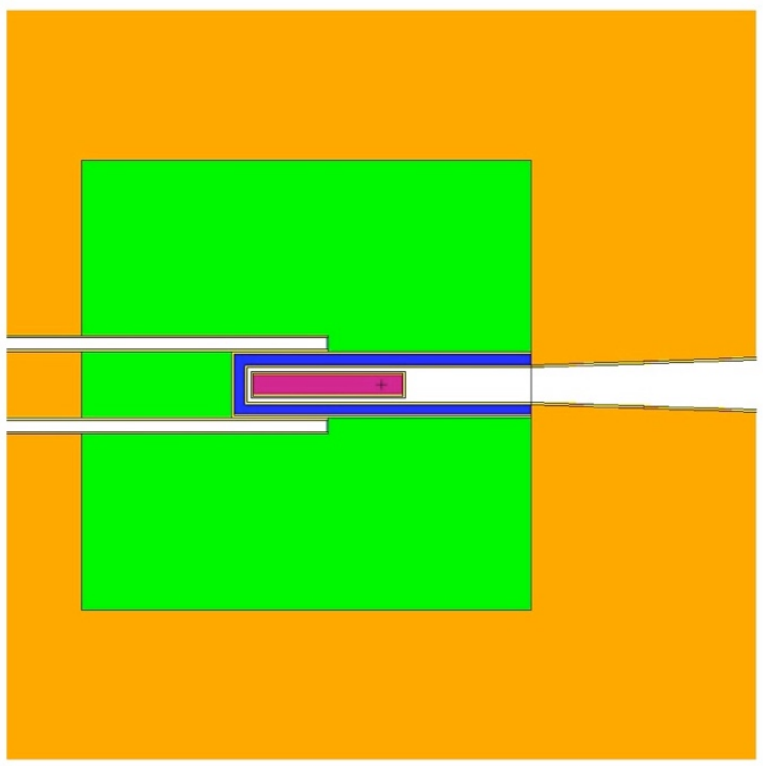

Fig. 2. 2-target geometry configuration.

\subsection{Neutron production}

Fig. 3 and Fig. 4 show maps of neutron fluences with $E<$ $0.4 \mathrm{eV}$ for the 1- and 2-target configurations, respectively. In both configurations, the density of neutrons is more 
concentrated at the center of the system, as expected. This has some implications on the design choice of the targetmoderator system, because the cold moderator should be placed where the thermal flux is maximum. This obviously favours a 2-target geometry since in this case the moderator is placed at the center of the system, and is more uniformly illuminated by thermal neutrons; a higher brightness is therefore expected from this geometry.

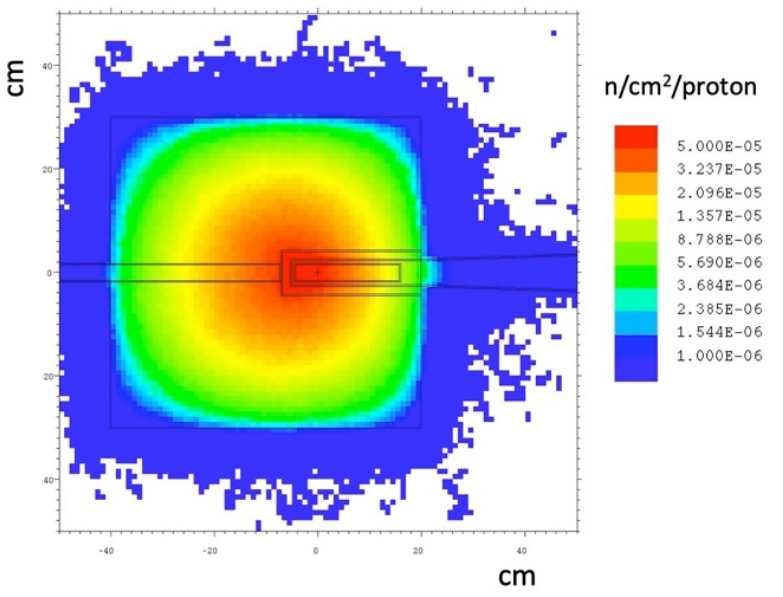

Fig. 3. Low energy $(E<0.4 \mathrm{eV})$ neutron fluence map for the 1target configuration.

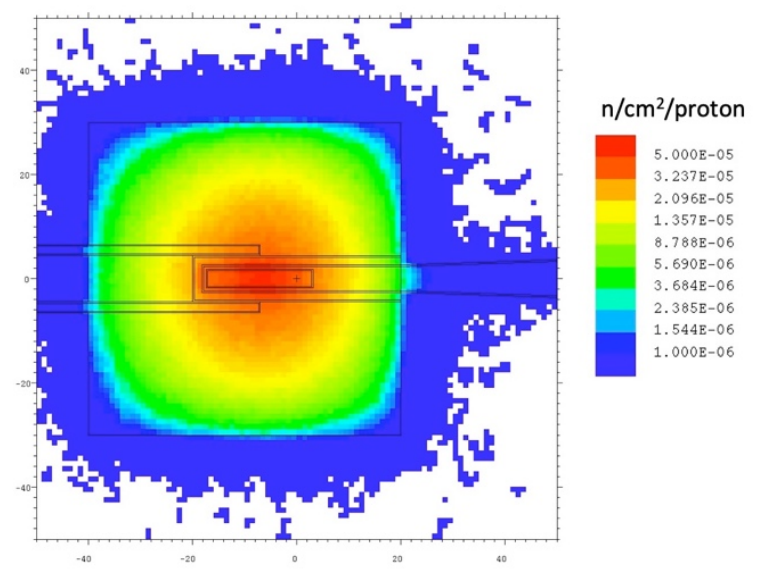

Fig. 4. Low energy $(E<0.4 \mathrm{eV})$ neutron fluence map for the 2target configuration.

\subsection{Moderator length}

The source performance for different moderator lengths is shown in Fig. 5 for the 1-target and 2-target configurations. For these calculations, a $1.5 \mathrm{~cm}$ thick water premoderator was assumed. In the case of the 1target geometry, the fact that moderator is not placed in the center limits the optimal moderator length to about 12 $\mathrm{cm}$. For longer moderator lengths, neutron absorption by hydrogen dominates and this reduces the performance of the moderator. On the contrary, for the 2-target configuration the optimal moderator length is of about $20 \mathrm{~cm}$. This confirms that a 2-target configuration is more suitable to be used with a tube moderator. In fact a moderator length of $12 \mathrm{~cm}$ is about equal to the mean free path of cold neutrons in pure parahydrogen $(11 \mathrm{~cm} \mathrm{[2]).}$ The optimal moderator length of a parahydrogen tube moderator should be considerably longer than that, if most of the moderator is uniformly illuminated by thermal neutrons; a 20-25 cm length is expected to be optimal, as confirmed by the ESS design [1]. Therefore, the 2-target geometry was adopted for the remaining neutronic studies presented in this paper.

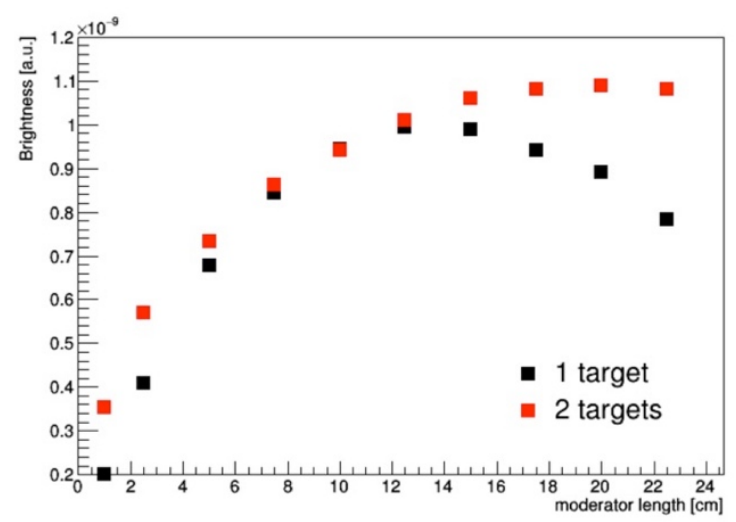

Fig. 5. Effect of moderator length on the source cold brightness (integral brightness below $5 \mathrm{meV}$ ) for the 1-target and 2-target geometries.

\subsection{Effect of premoderator}

We studied the effect of the presence of a water premoderator surrounding the cold moderator for the 2target geometry. Fig. 6 shows the thermal neutron maps without and with a premoderator $(1.5-\mathrm{cm}$ thick). It is clear that having a premoderator increases the concentration of thermal neutrons reaching the cold moderator. Fig. 7 shows the effect on the brightness: the optimal thickness of the premoderator is of $1.5 \mathrm{~cm}$. 


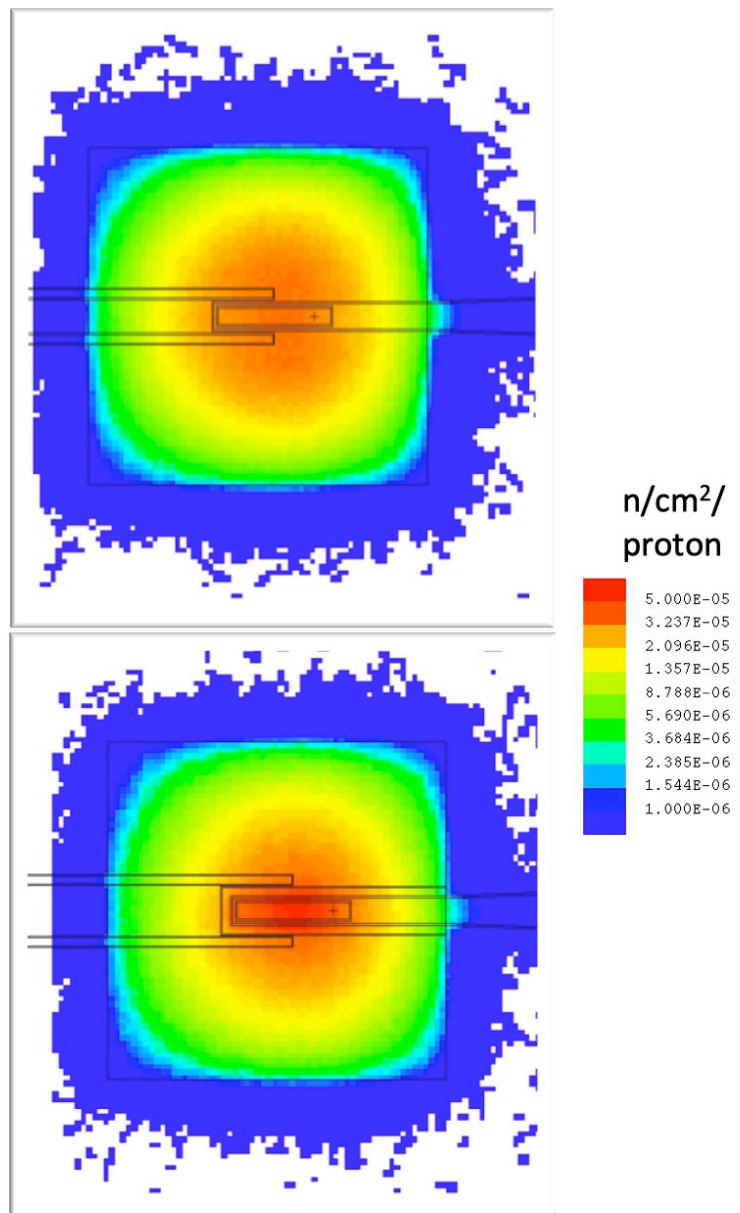

Fig. 6. Effect of the presence of premoderator on the thermal neutron fluence map $(E<0.4 \mathrm{eV})$ in the compact source. Top figure: no premoderator. Bottom figure: $1.5-\mathrm{cm}$ thick premoderator.

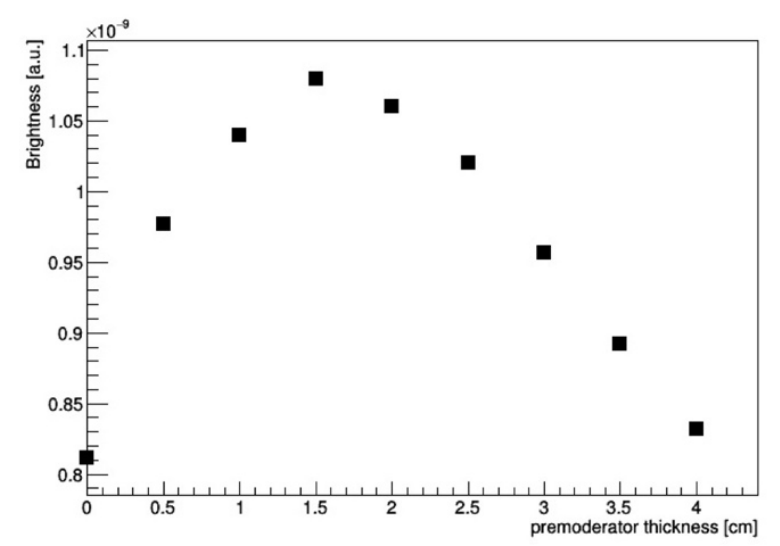

Fig. 7. Effect of premoderator thickness on the source cold brightness.

\subsection{Size of Be reflector}

Ideally the beryllium reflector surrounding the moderators should be as large as possible. For a compact source however we are interested in reaching an optimal compromise between size (cost) and performance. We considered the variation of the moderator brightness with the size (lateral dimension of a cube of beryllium), in the case with and without premoderator (Fig. 8). The brightness, relative to the maximum achievable with a 1$\mathrm{m}$ size reflector, changes more steeply with the size in the case without premoderator; this is due to the optimal shaping of the thermal neutron map that is achieved by using a premoderator. Fig. 8 indicates that a compact Be reflector with a size between 40 and $50 \mathrm{~cm}$ would deliver about $90 \%$ of the maximum performance obtained with a large reflector. We therefore see that the advantage of a water premoderator is two-fold: increase of brightness by optimal shaping of the neutron spectrum feeding the moderator, and decrease of the size of the Be reflector. To further reduce costs, it could be worthwhile considering a graphite or lead reflector as a cheaper alternative to beryllium.

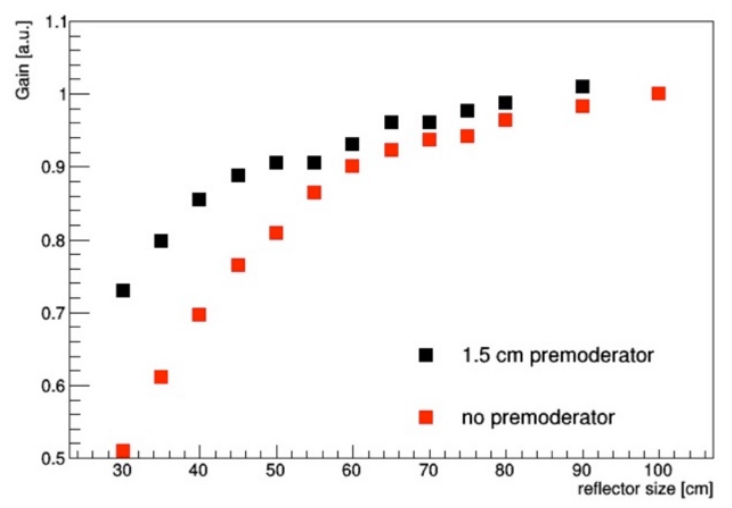

Fig. 8. Dependence of the source cold brightness on the size of Be reflector, with and without water premoderator.

\subsection{Size of targets}

The results shown so far are for (ideal) very small beryllium targets. However, for operation at power levels of the order of 50-100 kW, the surface of the neutronproducing target must be increased to allow for cooling of the target. Indications are that a surface of $100 \mathrm{~cm}^{2}$ would be required to allow operation at $100 \mathrm{~kW}$ (typically $1 \mathrm{~kW} / \mathrm{cm}^{2}$ is considered the upper limit for water cooling of a conventional flat target [9]). The increase in target size will result in a loss in performance, which is of about $30 \%$ at $100 \mathrm{~cm}^{2}$, compared to an ideal point-like target, see Fig. 9. The decrease is for both 1 and 2 target configurations, but the 2-target configuration remains better performing for all values of target surface. 


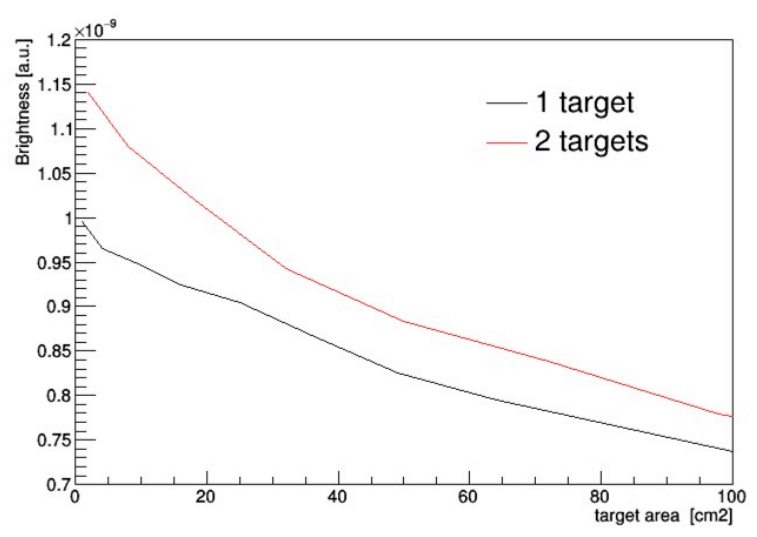

Fig. 9. Target size effect. See explanation in the text.

\section{Performance compared with high- power sources}

In order to compare the efficiency of two different types of neutron sources, i.e., high-power spallation and compact Be-target sources, we use as a figure of merit the brightness per fast neutron produced in the target. More specifically, as done in Ref. [3], we consider the yield $Y$ (number of neutrons per proton on target), which is of about $38 \mathrm{n} / \mathrm{p}$ for $2 \mathrm{GeV}$ protons on tungsten, and about $6.2 \times 10^{-3} \mathrm{n} / \mathrm{p}$ for the compact source at $13 \mathrm{MeV}$ [3]. The brightness $B(E<5 \mathrm{meV})$ for ESS is of about $1 \times 10^{-3}$ $\mathrm{n} / \mathrm{cm}^{2} / \mathrm{s} / \mathrm{sr} /$ proton, while for the compact sources is of about $1.1 \times 10^{-6} \mathrm{n} / \mathrm{cm}^{2} / \mathrm{s} / \mathrm{sr} /$ proton. We have therefore a ratio $B / Y$ of about $2.6 \times 10^{-5}$ for ESS, and $1.8 \times 10^{-4}$ for the compact source. This is for configuration of the compact source calculated for the 2-target design, with a target surface area of $100 \mathrm{~cm}^{2}$ (physics model with no engineering design). The results indicate that a small source with a tube moderator can be one order of magnitude more efficient in slowing down fast neutrons, than a high-power source like ESS. Part of the gain can be attributed to the choice of using a 1D tube moderator, which will allow to serve a smaller number of beamlines (up to 4-6 for each side of the moderator). Part of the gain is also due to the more compact system and the possibility to arrange in a more flexible way the moderator and reflector around the neutron producing target.

If we compare the source performances at operating power of $5 \mathrm{MW}$ for ESS, and (expected) $100 \mathrm{~kW}$ for the compact source, the brightness values will be of about $1.6 \times 10^{13} \mathrm{n} / \mathrm{cm}^{2} / \mathrm{s} / \mathrm{sr}$ for ESS, and about $5 \times 10^{10} \mathrm{n} / \mathrm{cm}^{2} / \mathrm{s} / \mathrm{sr}$ for the compact source, i.e., a ratio of about 300 . Even if this ratio is expected to increase when calculated for a fully engineered compact source, this level of performance is very promising for a variety of neutron scattering experiments that will not require extremely high fluxes like what is available at ESS.

\section{Conclusions}

The use of low-dimensional moderators is particularly suitable for compact neutron sources. Such compact systems allow the effective coupling of an optimized moderator and reflector system. The use of a premoderator allows the shaping of the thermal flux feeding the cold moderator, with a consequent increase in performance, as well as the reduction of the size of the reflector. The 2-target geometry that we investigated is particularly promising, and would be convenient for the cooling of the target, as the beam power is split into two targets. The efficiency of these sources, measured by the ratio of the moderator brightness to the number of fast neutrons produced by the target, can be one order of magnitude higher than high-power spallation sources. Thus, use of low-dimensional moderators in compact sources will reduce the gap with high-power sources, giving the possibility to perform high-quality neutron scattering measurements.

\section{References}

1. L. Zanini, K. H. Andersen, K. Batkov, E. B. Klinkby, F. Mezei, T. Schoenfeldt, A. Takibayev, Nucl. Instrum. Methods A 925, 33 (2019)

2. F. Mezei, L. Zanini, A. Takibayev, K. Batkov, E. Klinkby, E. Pitcher, T. Schoenfeldt, Journal of Neutron Research 17, 101 (2014).

3. L. Zanini, F. Mezei, K. Batkov. E. Klinkby, A, Takibayev, IOP Conf. Series: Journal of Physics: Conf. Series 1021, 012009 (2019).

4. K. H. Andersen, M. Bertelsen, L. Zanini, E.B. Klinkby, T. Schoenfeldt, P. M. Bentley, J. Saroun, JAC 51, 264 (2018).

5. C.M. Lavelle et al., Nucl. Instrum. Methods A 587 (2008) 324.

6. D. V. Baxter et al, Nucl. Instrum. Methods B 241, 209 (2005).

7. D. Pelowitz, editor, MCNPX user Manual, Version 2.7.0, LA-CP-11-0438 (2011).

8. L. S. Waters, et al., AIP Conf. proc. 896, 81 (2007).

9. D. V. Baxter, CANS TMR design: a personal reflection, presentation at UCANS-8, Paris, 2019. 